\title{
Development of a Practical Calibration Procedure for a Clinical SPECT/MRI System Using a Single INSERT Prototype Detector and Multi-Mini Slit-Slat Collimator
}

\author{
Debora Salvado, Kjell Erlandsson, Michele Occhipinti, Carlo Fiorini, Jurgen Willems, Pieter van Mullekom, \\ Zoltan Papp, Miklos Czeller, Kalman Nagy and Brian F. Hutton
}

\begin{abstract}
In the context of the INSERT project, we have been developing a clinical SPECT insert for an MRI system, in order to perform simultaneous SPECT/MRI of the human brain. This system will consist of 20 CsI:Tl scintillation detectors, $5 \mathrm{~cm}$ wide and $10 \mathrm{~cm}$ long, with a 72-channel SiPM readout per detector, and a multi-mini slit-slat (MSS) collimator set up in a stationary partial ring. Additionally the system has a custom-built transmit/receive MR coil to ensure compatibility with the SPECT system. Due to the novel design of the system/collimator, existing geometric calibration methods are not suitable. Therefore we propose a novel and practical calibration procedure that consists of a set of specific independent measurements to determine the geometric parameters of the collimator. This procedure was developed utilising a prototype system that consists of a reducedsize single detector with a 36-channel SiPM-based readout and a single MSS collimator module. Validation was performed by reconstructing different imaging phantoms, using a rotating stage to simulate a tomographic acquisition. Regarding uniformity, the COV for the cylinder phantom reconstructed with correct calibration parameters is $6.7 \%$, whereas the $\mathrm{COV}$ using incorrect parameters is $9.4 \%$. The quality of the phantom reconstructions provide evidence of the applicability of the proposed method to the calibration of the prototype system. This procedure can be easily adapted for the final INSERT system.
\end{abstract}

Index Terms-practical calibration, prototype system, INSERT.

\section{INTRODUCTION}

$\mathbf{I}$ $\mathrm{N}$ the context of the INSERT project [1], we have been developing a Single Photon Emission Computed Tomography (SPECT) insert for a Magnetic Resonance Imaging (MRI) system, in order to perform simultaneous SPECT/MRI of the human brain. Within the project, the goal is to provide enhanced stratification in glioma patients, but a simultaneous SPECT/MR system could be beneficial in other areas of neurological imaging [2], [3], [4]. The system will be based on MRcompatible detectors [5] with 8 mm-thick CsI:Tl scintillation

D. Salvado, K. Erlandsson and B. F. Hutton are with the Institute of Nuclear Medicine, University College London, UK. B. F. Hutton is also with the Centre for Medical Radiation Physics, University of Wollongong, Australia M. Occhipinti and C. Fiorini are with Politecnico di Milano, Dipartimento di Elettronica, Informazione e Bioingegneria, Italy, and the INFN, Sezione di Milano, Italy. J. Willems and P. van Mullekom are with Nuclear Fields, Vortum-Mullem, The Netherlands. Z. Papp, M. Czeller and K. Nagy are with Mediso Medical Imaging Systems, Budapest, Hungary.

Corresponding author: Débora Salvado, debbiesal@gmail.com.

Manuscript received August 1, 2017; revised November 24, 2017; revised February 15, 2018; revised March 25, 2018; accepted March 27, 2018.

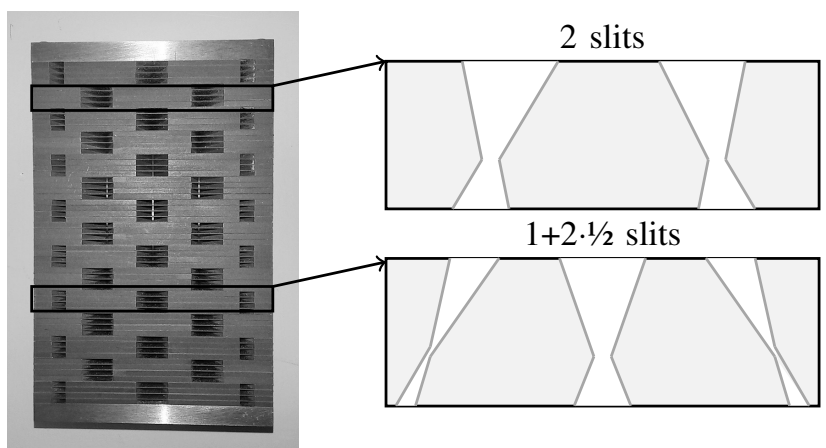

Fig. 1. Picture of the prototype MSS collimator (left) and illustration of the transaxial collimation by the different mini-slit sections (right), the 2 -slit and the $1+2 \cdot 1 / 2$-slit sections.

crystals and silicon photomultiplier (SiPM) readout with a size of $10 \times 5 \mathrm{~cm}^{2}$ [6], [7]. The good intrinsic resolution of $1 \mathrm{~mm}$ [8] supports minification, which is obtained by using a collimator-detector distance smaller than the collimator-patient distance. This enables the design of a compact and highsensitivity collimator based on slit-slat collimation, the multimini slit-slat (MSS) collimator [9], [10], [11], [12]. In contrast to a conventional multi-slit-slat collimator, the MSS collimator has arrays of mini-slits, i.e. short sections of the slit component, instead of a continuous slit aperture, and each mini-array is misaligned with the adjacent one. Therefore, along the axial direction, there are two interspersed sections: one with 2-minislit apertures and one with $1+2 \cdot 1 / 2$-mini-slit apertures. A halfslit is an aperture shared by adjacent detectors in the ring configuration. Finally, the slit aperture is interior to the slat component, allowing the slats to extend beyond the slit plane (Figure 1). The final INSERT design consists of a stationary partial ring of twenty detectors coupled to an MSS collimator, with an angular coverage of 285 degrees, and a customised transmit/receive RF coil to image a cylindrical FOV of $20 \mathrm{~cm}$ diameter and $9 \mathrm{~cm}$-height.

Calibration is essential to obtain a correct correspondence between the manufactured system geometry, and that modelled during reconstruction through the system matrix, in order to ensure optimal reconstruction, avoiding the presence of artefacts and loss of resolution of the reconstructed images [13]. The system matrix depends on the properties 
of the object, image system and detector. Therefore, system calibration includes a number of corrections that account for detector linearity, energy linearity, collimator efficiency, and the system's geometry and point spread function (PSF).

Geometric calibration can be achieved by scanning a collimated source to obtain the detector response and a point source in a fine grid through the whole FOV, with enough counts, to obtain the system matrix [14]. Due to the long acquisition times, different approaches have been suggested based on the measurement of the point response function (PRF) in a limited number of voxel positions, and interpolation for the whole FOV [15], [16], [17], [18]. Although these methods are highly accurate and especially well-suited for stationary systems, they assume a slowly varying PRF and they require long scanning times and sophisticated positioning tools.

On the other hand, model-based calibration methods rely on the modelling of the system response as a function of a set of geometric parameters. These parameters are then estimated minimising a cost function that compares experimental projection data with that obtained via an analytical model of the system response. This type of calibration is a more flexible method, but a good accuracy of the estimated parameters is sometimes hard to achieve due to correlations between parameters. For pinhole cameras, it has been proposed that the geometry of the pinhole can be fully described by seven parameters assuming a circular orbit of the camera. These parameters are focal length, distance $d$, mechanical offset, electrical shifts (two), and twist and tilt angles. Using the centre of mass of the projection of three non-collinear point sources, the calibration parameters are calculated by a least squares fit of the estimated point source projection locations to the measured mass centres [19], [20]. For large rotating cameras, deviations from the circular orbit can occur, but only a subset of the parameters related to the displacement of the camera needs to be recalculated [21]. In the case of slitslat collimators, calibration requires two separate minimisation procedures, one transaxially for the slit position, the centre of rotation (COR) and the starting position of the rotating point source relative to the COR, and one axially for the tilt and twist angles of the slats, using projection data from a rotating phantom with two point sources [22], [23], [24], [25].

In the case of the INSERT system, the clinical setting and the novel design of the collimator and system pose constraints to the calibration procedure. The detector response of the MSS collimator is discontinuous across the FOV, because each aperture does not cover the entire FOV and the slits are not continuous. Therefore, the grid for the scanning point source method would need to be very fine, especially in the presence of cameras with minification [26], and it would require an interpolation between the scanning points assuming a smooth variability in the point response function. This procedure is not practical, as it would take too long. On the other hand, a model-based calibration method would be more flexible and easier to adapt to the final INSERT system, with the possibility of verifying it inside the MR system.

Our aim was to develop a practical calibration procedure that can be easily translated to the final INSERT system. Taking advantage of the different types of collimation in the

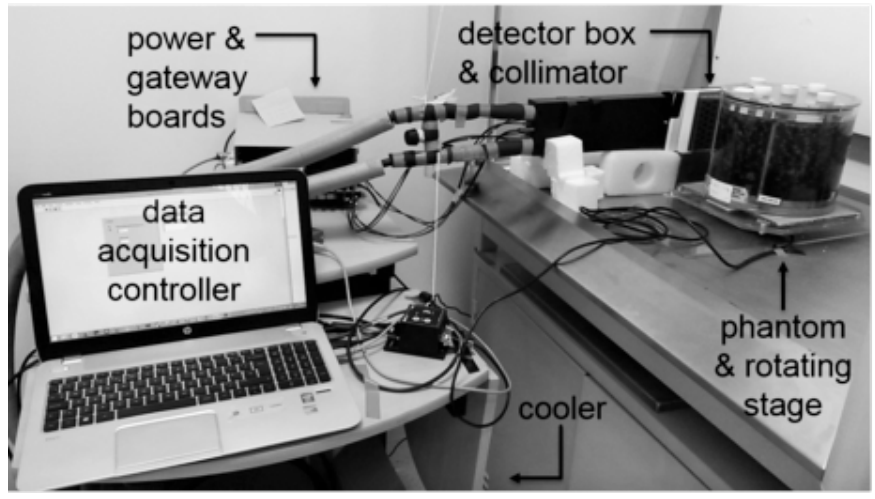

Fig. 2. Main setup of the INSERT prototype system with a single $5 \times 5 \mathrm{~cm}^{2}$ detector and a prototype MSS collimator.

transaxial and axial directions of the MSS collimator, we propose a combined-procedure with separate measurements to directly measure some calibration parameters and estimate others based on existing models [19], [22]. For that, we use a single-detector INSERT prototype system to develop and validate a practical calibration procedure.

\section{MethodS}

For the INSERT prototype system, we utilised a reducedsize single detector. The main setup of this system consisted of a plastic black box with the $5 \times 5 \times 0.8 \mathrm{~cm}^{3}$ scintillation crystal mounted on a 36-channel SiPM array, a liquid heat sink, and electronic boards for signal filtering and communication. The heat sink was connected to a cooling system that recirculates liquid at $-5{ }^{\circ} \mathrm{C}$, for improved performance of the SiPMs. The detector box was powered and connected to a computer through a gateway board and Ethernet cable. The crystal side of the detector box was closely aligned with a tungsten MSS prototype collimator, and lead blocks were positioned around it to shield the crystal. The parameters of the prototype MSS collimator are the following: $1.5 \mathrm{~mm}$ of slat spacing, $0.25 \mathrm{~mm}$ of slat thickness, $0.5 \mathrm{~mm}$ of slit aperture, and a mini-slit axial length of $6 \mathrm{~mm}$. Finally, a source of activity was positioned within the FOV for a static scan, or on top of a rotating stage to simulate a tomographic acquisition. An example of an acquisition setup is indicated in Figure 2.

The scanning parameters, such as total acquisition time and number of rotations/views, were controlled via computer interfaces for the detector and controller of the rotating stage. Acquired events were transformed into planar projection data using a maximum likelihood (ML) algorithm with a forward model based on [27]. This event-position reconstruction method utilises a ML algorithm to compute the position and energy of detected events by maximising the likelihood of obtaining the acquired data, given a previously-generated light distribution model. The light distribution model consists of a set of Light Response Functions (LRFs), one per readout channel. These LRFs are generated by fitting a Gaussian function to each channel data, from a flood acquisition. Data from the $5 \mathrm{~mm}$ around the edges of the detector are discarded due to the poor resolution, i.e. usable FOV is $4 \times 4 \mathrm{~cm}^{2}$. 
TABLE I

ACQUISITION PARAMETERS FOR THE CALIBRATION PHANTOM SCANS PERFORMED WITH ${ }^{99 \mathrm{M}} \mathrm{TC}$.

\begin{tabular}{lccc}
\hline Phantom & $\begin{array}{c}\text { Activity per } \\
\text { Source }[\mathrm{MBq}]\end{array}$ & $\begin{array}{c}\text { Acquisition Time } \\
\text { per Scan }[\mathbf{s}]\end{array}$ & $\begin{array}{c}\text { Nb. of Views } \\
\text { over 360 }\end{array} \mathbf{3 0}^{\mathbf{2}}$ ] \\
\hline uniform planar & 70.0 & 18000 & 1 \\
capillary $_{\|}$ & 15.0 & 150 & 1 \\
capillary $_{\perp}$ & 17.5 & 1800 & 30 \\
\hline
\end{tabular}

Event data were energy-windowed and corrected for detector linearity (Section II-A and II-B). After geometric calibration (Section II-C), reconstruction of tomographic data was performed with the maximum-likelihood expectationmaximization (MLEM) algorithm based on angular blurring [28] (Section II-D). Attenuation was included in the system matrix, and scatter was taken into account by using a reduced $\mu$-value of $0.09 \mathrm{~cm}^{-1}$. Finally, data were analysed in order to obtain evidence of the performance of the proposed calibration procedure for the prototype system.

\section{A. Energy Calibration}

Data from five different radionuclides, ${ }^{99 \mathrm{~m}} \mathrm{Tc},{ }^{57} \mathrm{Co},{ }^{75} \mathrm{Se}$, ${ }^{177} \mathrm{Lu}$ and ${ }^{111} \mathrm{In}$, were acquired with a small source positioned at around $50 \mathrm{~cm}$ from the detector. Energy spectra were obtained from the flood acquisitions, as a function of the cumulative channels' signal. Each photopeak was fitted with a Gaussian function in order to estimate peak location. Finally, an energy-channel calibration curve was determined by linear regression.

\section{B. Linearity Correction}

Thanks to the high intrinsic spatial resolution of the detector module, we were able to use a parallel-hole collimator as a linearity phantom, irradiating the detector with a ${ }^{99 \mathrm{~m}} \mathrm{Tc}$ point source at a distance of $\sim 50 \mathrm{~cm}$, using an image matrix size of $256 \times 256$ and a pixel size of $0.2 \times 0.2 \mathrm{~mm}^{2}$. This parallelhole collimator was a high-resolution collimator with a holediameter of $2 \mathrm{~mm}$, a septa-thickness of $0.25 \mathrm{~mm}$ and a holelength of $40 \mathrm{~mm}$.

Focusing on the planar projection data from the central $40 \times 40 \mathrm{~mm}^{2}$ area, which corresponds to the useful FOV, we utilised a matrix of $20 \times 18$ control points to derive the $2 \mathrm{D}$ spatial transformation that maps the centroid positions estimated from the projection data to the corresponding physical hole positions.

In order to correct for detector linearity distortions, the derived transformation was applied to the acquired planar projection data using MATLAB (The MathWorks, Inc., Natick, Ma, USA) standard functions.

In order to evaluate the method, we analysed the transformed projection visually, and we calculated the mean and standard deviation of the distance between the projection centroids and the true hole positions.

\section{Geometric Calibration}

(a)
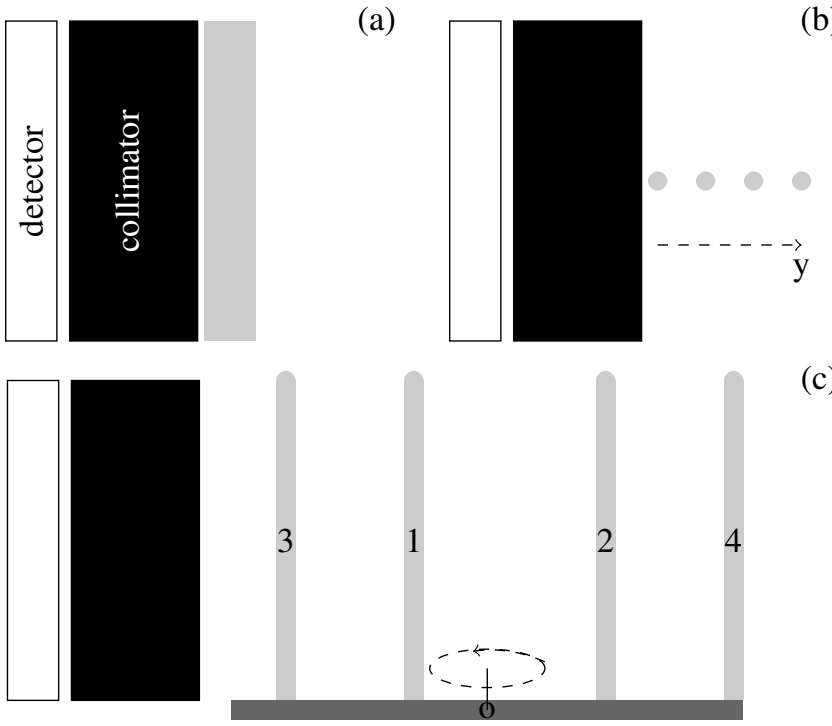

(c)

Fig. 3. Illustration of the different calibration phantoms: (a) planar source phantom for static acquisition, (b) capillary $y_{\|}$phantom for acquisition of the horizontal capillary at one position at a time, as indicated by the arrow, and (c) rotating capillary $\perp$ phantom. The vertical capillaries numbered 1-4 in the capillary $\perp$ phantom are positioned at a radius of 25, 50, 75 and $100 \mathrm{~mm}$ from the system COR, respectively.

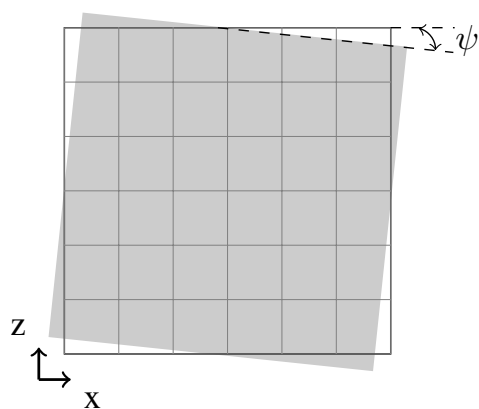

(a)

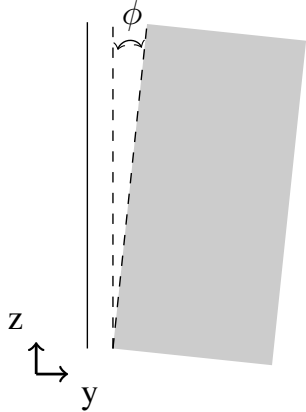

(b)

Fig. 4. Illustration of the twist (a) and tilt (b) calibration parameters. The grey shape corresponds to the collimator and the grid, to the detector plane.

We developed a framework that computes parameters to model the system response according to its geometry, based on pinhole calibration [19]. Instead of computing the parameters with a single procedure with a set of point sources, due to the specific design of the collimator, calibration is performed with three separate measurements to determine the geometric efficiency, the transaxial geometric parameters and the axial parameters, using three different calibration phantoms. The uniform phantom is a planar source $10 \mathrm{~cm}$-wide, $10 \mathrm{~cm}$-tall and $0.5 \mathrm{~cm}$-thick (Figure $3-\mathrm{a}$ ). The capillary $\|$ phantom has one capillary tube placed horizontally in the FOV (Figure 3-b). The capillary $\perp$ phantom has the capillary tubes placed vertically in the FOV; capillaries are positioned at radii of 25, 50, 75 and $100 \mathrm{~mm}$ from the centre of rotation (Figure 3-c). Each capillary tube has an internal diameter of $1.15 \mathrm{~mm}$ and a length of $75 \mathrm{~mm}$. For each phantom, the acquisition parameters are shown in Table I: for the uniform phantom, this is positioned as close as possible to the MSS collimator for a static scan; 
for the capillary $\|$ phantom, the capillary tube is acquired at eleven distances from the collimator, moving it away from the system $20 \mathrm{~mm}$ each time; and for the capillary $\perp$ phantom, data are acquired at different angles over $360^{\circ}$ by rotating the phantom around the system's COR.

In order to obtain the calibration parameters, we process the projection data as described below:

- determination of the geometric efficiency - The sensitivity profile per slit section of the collimator was determined from the planar projection data of the uniform phantom acquisition. This phantom has to be wider than the collimator to account for the half-slits.

- determination of the electronic shifts $e_{u}$ and $e_{v}$ - These shifts are calculated comparing the detector FOV centre to the projection data centre for the uniform phantom acquisition, defined as the centre of the collimator pattern in the projection image.

- determination of the twist $\psi$ - The twist is the angle between the slits and the $z$-axis of the object frame of reference (Figure 4-a). Therefore, the twist is given by the deviation of the capillary projection from the horizontal orientation, when imaging the capillary ${ }_{\|}$phantom.The twist angle depends on the relative alignment of the capillary, collimator and detector. We assume the capillary to be perfectly aligned with the horizontal plane, and the detector coordinates to be corrected for intrinsic linearity, as described above.

- determination of the tilt $\theta$ - The tilt is the angle between the slats and the $x y$-plane of the object frame of reference (Figure 4-b). The tilt is given by the amplitude of the up or down motion of the capillary projection as the source moves away from the collimator, by imaging the capillary $_{\|}$phantom with the capillary source at different distances from the collimator. The tilt angle is calculated as follows (Equation 1):

$$
\tan \theta=\frac{\Delta z}{\Delta R}
$$

where $z$ is the vertical detector coordinate, and $R$ the horizontal distance of the capillary from the detector.

- determination of the focal length $f$, mechanical shift $m$ and slit positions $s_{\mathrm{n}}$ - These transaxial parameters were estimated by optimizing the squared difference between the measured projection data and the analytically estimated projections, assuming the ROR (and therefore parameter $d$ ) is known to reduce the uncertainty of the focal length estimation. This is a reasonable assumption as the system is stationary. Projection data are acquired rotating the capillary $\perp$ phantom.

Due to the acquired capillary projection data at different distances from the crystal utilising the capillary $\|$ phantom, the axial resolution can also be determined as the full width at half maximum (FWHM) of the Gaussian fit of those projections in the axial direction, after correction for the finite width of the capillary. This is an important parameter, because it determines the overlap of the FOV for the different mini-slit sections in the object domain, thus defining the axial and angular sampling
TABLE II

CORRECT AND INCORRECT SET OF CALIBRATION PARAMETERS OF THE INSERT PROTOTYPE SYSTEM.

\begin{tabular}{lrrr}
\hline Parameter & Correct & Incorrect & Delta \\
\hline$f[\mathrm{~mm}]$ & 20.56 & 21.03 & 0.47 \\
$d[\mathrm{~mm}]$ & 172.25 & 172.25 & 0 \\
$m[\mathrm{~mm}]$ & 2.77 & 0.75 & -2.02 \\
$e_{u}[\mathrm{~mm}]$ & -0.92 & 1.46 & 2.38 \\
$e_{v}[\mathrm{~mm}]$ & 0.24 & 0.05 & -0.20 \\
$\theta\left[^{o}\right]$ & 0 & 0 & 0 \\
$\psi\left[{ }^{o}\right]$ & 0 & 0 & 0 \\
$s_{\mathrm{n}}[\mathrm{mm}]$ & 12.97 & 13.15 & 0.18 \\
& 21.68 & 21.98 & 0.30 \\
\hline
\end{tabular}

of the system. An incorrect value of this parameter can lead to artefacts in the reconstructed images.

Finally, all the aforementioned parameters are included in the reconstruction algorithm to achieve a proper calibration.

\section{Tomographic Experiments}

In order to test the proposed calibration procedure, we performed tomographic experiments with three different imaging phantoms: uniform cylinder, spheres and Hoffman brain [29] phantoms. Each phantom was filled with a specific ${ }^{99 m} \mathrm{Tc}$ activity and positioned at the centre of the FOV, on top of the rotating platform to acquire 25 views over $360^{\circ}$. The distance between the COR and the collimator surface was set to $165 \mathrm{~mm}$, so as to represent the geometry of the full INSERT system, according to the patient aperture between collimator surfaces. We had to perform long acquisition scans due to the fact that it was only one small detector: 500, 100 and 450 minutes for the uniform, spheres and Hoffman phantom, respectively, which correspond to total counts of $1.50 \mathrm{M}$, $1.95 \mathrm{M}$ and $483 \mathrm{k}$, for the respective phantoms. Raw data were transformed into planar projections using an energy window of $140 \mathrm{keV} \pm 15 \%$. These projections were corrected as mentioned in the previous sections and reconstructed with 40 MLEM iterations. The calibration parameters were accounted for in the reconstruction algorithm. Attenuation was assumed to be uniform in the cylindrical phantoms, but was not included for the spheres.

The uniform phantom consisted of a cylindrical phantom with an inner diameter of $184 \mathrm{~mm}$, filled with water up to $20 \mathrm{~mm}$ height, with $69.1 \mathrm{MBq}$ of ${ }^{99 m}$ Tc activity. Two reconstructions were performed, one utilising the estimated calibration parameters and a second where calibration parameters from a different day, in which the collimator was repositioned relative to the detector, were used to investigate the effect on the reconstruction (Table II); no tilt and twist calibrations were performed on these days. For the reconstruction with correct calibration, intensity profiles were determined along different directions of the transaxial plane. After summing axially the reconstructed image slices for $14.3 \mathrm{~mm}$, we calculated the coefficient of variation (COV) as the standard deviation divided by the mean over a circular ROI of diameter $160 \mathrm{~mm}$.

The spheres phantom consisted of a cylindrical phantom with fillable sphere inserts in air. There were two sets of spheres with $1.7 \mathrm{~cm}, 1.4 \mathrm{~cm}$ and $1.1 \mathrm{~cm}$ diameters and a single sphere with a diameter of $2.1 \mathrm{~cm}$. An initial volume 


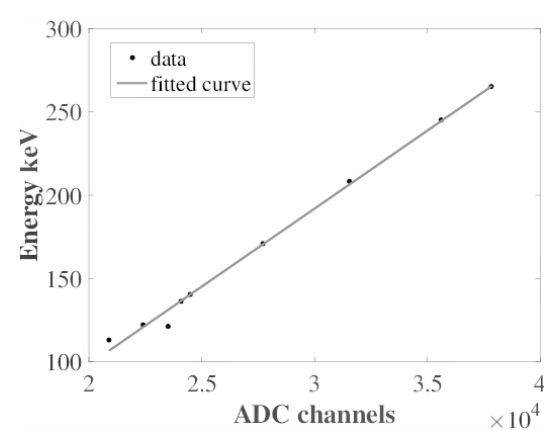

Fig. 5. Energy-channel calibration curve. Each dot corresponds to a photopeak measurement and the line to the fitted curve.
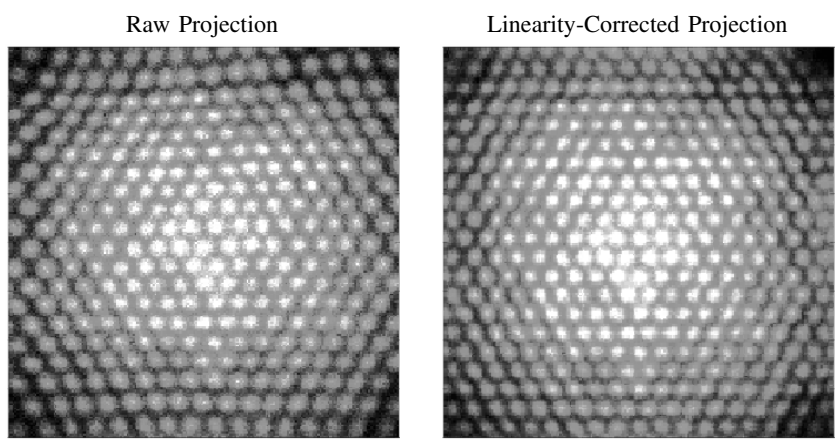

Fig. 6. Planar projection data obtained with a parallel-hole collimator and a point source at a distance: raw data (left) and linearity-corrected data (right).

of $5.8 \mathrm{ml}$ was prepared with $51.3 \mathrm{MBq}$ of ${ }^{99 m} \mathrm{Tc}$ activity. The first set of three spheres was filled first. The remaining solution was diluted with water, reducing the concentration to half of the previous value. Then the second set of three spheres was filled, and the remaining solution was diluted again to half of the previous value. Finally, the last sphere, i.e. the biggest sphere, was filled. Hence, the sets of spheres had a relative activity concentration of $4: 2: 1$. After reconstruction, each sphere activity concentration was calculated as the sum of the intensities in a 3D region containing the reconstructed sphere divided by the true sphere volume.

Finally, the Hoffman phantom was filled with $110.4 \mathrm{MBq}$ of ${ }^{99 m} \mathrm{Tc}$ activity, scanned and reconstructed to qualitatively assess the activity distribution.

\section{RESULTS}

\section{A. Energy Calibration}

Figure 5 shows the energy-channel calibration curve for the prototype INSERT detector, using the photopeak data of different radionuclides. The coefficient of determination is very close to 1, demonstrating a good fit between the cumulative channel signal and the energy, i.e. a good detector energy linearity. Therefore the energy-channel calibration curve was subsequently used to select recorded events at a specific energy window.

\section{B. Linearity Correction}

Regarding the detector's spatial linearity, Figure 6 shows linearity-corrected and uncorrected planar projection data of
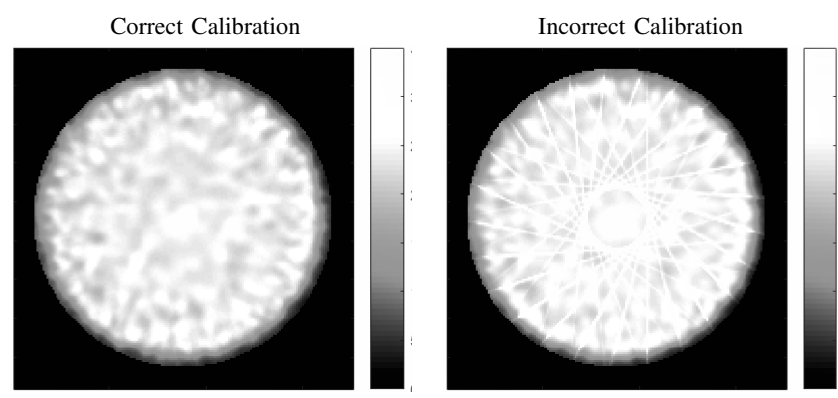

Fig. 7. Transaxial view of the sum of the central slices of the reconstructed image of the uniform phantom, using the correct (left) and incorrect (right) geometric calibration parameters during reconstruction.

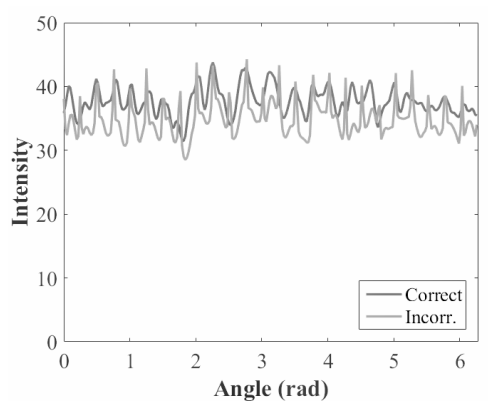

Fig. 8. Circular profiles for both the correctly and incorrectly calibrated reconstructions of the uniform phantom.

the measurements with the parallel-hole collimator and a distant radioactive point source. In comparison to the uncorrected planar projection image, the linearity-corrected one shows a reduction of image distortion, especially at the borders of the image. Regarding the distance between the control points, i.e. uncorrected centroids and true hole positions, the mean displacement is $1.05 \mathrm{~mm}$ and the standard deviation is $0.75 \mathrm{~mm}$.

\section{Tomographic Experiments}

Applying the proposed calibration procedure, a set of geometric parameters was obtained and applied in the reconstruction of the tomographic acquisitions.

Figure 7 shows the transaxial view of the average of the central slices of the cylindrical phantom reconstruction utilis-
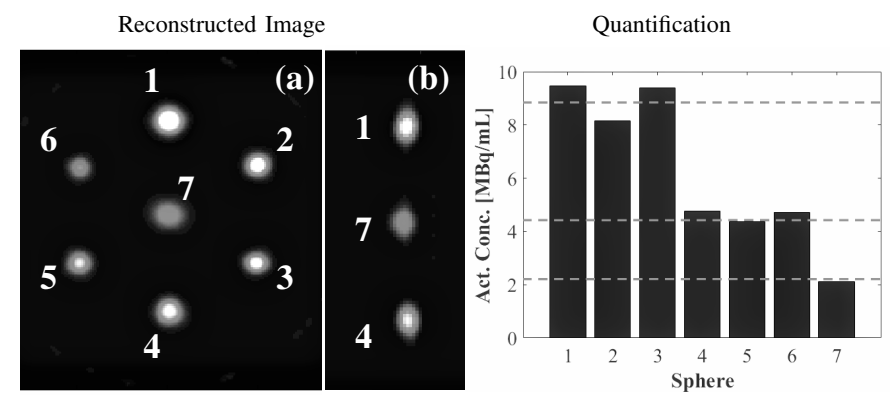

Fig. 9. Central slice of the reconstructed spheres phantom (left) - transaxial (a) and axial (b) views, and corresponding estimated activity concentrations (right). The dashed lines correspond to the true activity concentrations. Spheres 1 to 7 have diameters of $17,14,11,17,14,11,21 \mathrm{~mm}$, respectively. 


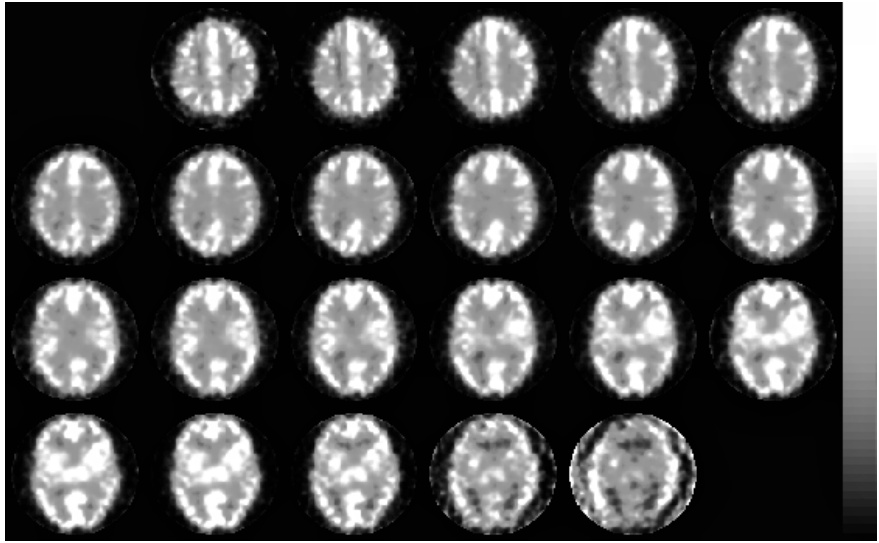

Fig. 10. Axial slices of the Hoffman brain phantom reconstruction.

ing the determined calibration parameters (left) and incorrect calibration parameters (right). When applying the incorrect calibration parameters, streak and ring artefacts appear in the reconstructed image. The $\mathrm{COV}$ for the image reconstructed with correct calibration parameters is $6.7 \%$, whereas the COV using incorrect parameters is $9.4 \%$. In addition, Figure 8 shows circular profiles, at a radius of $63 \mathrm{~mm}$, for both the correctly and incorrectly reconstructed image of the uniform phantom acquisition. When using the incorrect calibration parameters, the average intensity reduced and multiple peaks due to the streak artefacts can be seen.

Figure 9 shows transaxial and sagittal views of the reconstructed image for the spheres phantom acquisition. Each sphere has the expected shape and position. In addition, the bar plot of Figure 9 shows the estimated activity concentration for each sphere. For spheres 1-3, 4-6 and 7, the dashed lines correspond to the true activity concentrations values. Each sphere estimated activity concentration is close to the true value. The mean absolute deviation from true values was $5.8 \%$ (SD $2.7 \%$ ).

Finally, Figure 10 shows a set of axial slices with transaxial views of the reconstructed image for the Hoffman brain phantom acquisition. The activity distribution within the brain is similar to that obtained with a conventional SPECT system. Note that the whole brain is not shown in the reconstructed images because the brain is larger than the detector's FOV in the axial direction.

\section{DISCUSSION}

Energy-channel linearity was very good, allowing for the correct energy windowing of the acquired data.

Regarding the detector's linearity, the improvement in image quality suggests that an appropriate spatial transformation was derived for the correction. Quantitatively, the distortions were small, but the correction clearly improves the linearity, especially at the edges of the detector's FOV. Note that this experiment was only possible due to the high intrinsic spatial resolution of the prototype detector.

For the tomographic experiments, the ability to reconstruct images utilising the acquired data validate the proposed calibration procedure for the prototype system. Furthermore, for the example of the uniform phantom reconstruction using incorrect calibration parameters, the presence of artefacts indicates the importance of a good system calibration to ensure optimal reconstruction.

In the case of the uniform cylindrical phantom acquisition, despite the presence of noise due to a low count-level, results indicate a reconstructed image without major artefacts. For the spheres phantom acquisition, each sphere is well reconstructed, and quantification of the activity concentrations are in good agreement with the relative set activity concentrations. In the sagittal view, the spheres appear slightly elongated; this needs further investigation. For the Hoffman brain phantom acquisition, results indicate the prototype system's ability to image a realistic activity distribution.

Finally, the proposed practical calibration procedure can be easily adapted for the final INSERT system, but some details of the practical implementation may need to be modified for geometrical reasons. We envisage an extended planar source placed $1 \mathrm{~cm}$ from the collimator, and a capillary-parallel phantom with three line sources placed at the centre of the FOV, so that each detector can be calibrated on the constructed system. Given the time constraints and the fact that it is a stationary system, we plan to calibrate the standalone SPECT system outside the MR room, and then perform only one measurement mounted in the MR system with line sources to ensure that the calibration is correct. In this scenario the capillary-perpendicular phantom can be rotated manually with high precision.

Although the objective of this paper was to develop a practical calibration procedure, note that the final INSERT system has a partial ring design, so the absence of detectors should limit the spatial resolution of the reconstructed image in the lower part of the FOV corresponding to the region close to the detector gap in contrast to the results shown here, which were obtained for a complete ring geometry [12]. On the other hand, the detector's production technology has been improved, so the final INSERT system will have detectors with better overall performance. As future work, we are planning to conduct a full system performance evaluation, with the INSERT operating as a SPECT-alone system, and with the INSERT inside the MRI system.

\section{CONCLUSION}

Precise calibration of a SPECT camera is essential to obtain a good-quality image reconstruction. Due to the unique system and collimator design, we have developed a practical calibration framework with independent measurements to obtain calibration parameters, using a prototype INSERT system. Reconstruction of tomographic acquisitions of different imaging phantoms provide evidence of the efficacy of the proposed method. This model-based calibration procedure can then be easily adapted to the final INSERT system.

\section{ACKNOWLEDGMENT}

The authors would like to acknowledge support from the PhD Fellowship SFRH/BD/88093/2012 by Fundação para a Ciência e Tecnologia, Portugal; the INSERT collaboration, 
which is funded by the European Commission under FP7HEALTH-305311; and the National Institute for Health Research, University College London Hospitals Biomedical Research Centre.

\section{REFERENCES}

[1] About the INSERT Project. [Online]. Available: http://www.insertproject.eu/insert/about-insert

[2] R. T. M. de Rosales, "Potential Clinical Applications of Bimodal PET-MRI or SPECT-MRI Agents," Journal of Labelled Compounds and Radiopharmaceuticals, vol. 57, no. 4, pp. 298-303, 2014. [Online]. Available: http://dx.doi.org/10.1002/jlcr.3154

[3] V. Sulc, S. Stykel, D. P. Hanson, B. H. Brinkmann, D. T. Jones, D. R. Holmes, R. A. Robb, M. L. Senjem, B. P. Mullan, R. E. Watson et al., "Statistical SPECT Processing in MRI-Negative Epilepsy Surgery," Neurology, vol. 82, no. 11, pp. 932-9, 2014.

[4] M. R. Bernsen, P. E. B. Vaissier, R. Van Holen, J. Booij, F. J. Beekman, and M. de Jong, "The Role of Preclinical SPECT in Oncological and Neurological Research in Combination with Either CT or MRI," European Journal of Nuclear Medicine and Molecular Imaging, vol. 41, no. 1, pp. 36-49, May 2014. [Online]. Available: https://doi.org/10.1007/s00259-013-2685-3

[5] G. L. Montagnani, M. Occhipinti, M. Carminati, and C. Fiorini, "Mapping Tool for Investigation of Component-Level PCB Compatibility in Multimodal MRI/SPECT," in 2016 IEEE NSS/MIC, Nov. 2016.

[6] P. Busca, C. Fiorini, A. D. Butt, M. Occhipinti, R. Quaglia, P. Trigilio, K. Nagy, T. Bukki, M. Czeller, Z. Nyitrai, C. Piemonte, A. Ferri, A. Gola, and J. Rieger, "Development of a SiPM-Based Anger Camera for INSERT, a New Multi-Modality SPECT/MRI System for Preclinical and Clinical Imaging," in 2014 IEEE NSS/MIC, Nov. 2014.

[7] M. Occhipinti, P. Busca, A. D. Butt, G. Cozzi, C. Fiorini, I. Perali, F. Acerbi, A. Ferri, A. Gola, and C. Piemonte, "A Compact SiPM Photodetector Array for SPECT Applications," in 2014 IEEE NSS/MIC, Nov. 2014.

[8] P. Busca, M. Occhipinti, P. Trigilio, G. Cozzi, C. Fiorini, C. Piemonte, A. Ferri, A. Gola, K. Nagy, T. Bukki, and J. Rieger, "Experimental Evaluation of a SiPM-Based Scintillation Detector for MR-Compatible SPECT Systems," IEEE Transactions on Nuclear Science, vol. 62, no. 5, pp. 2122-8, Oct. 2015.

[9] D. Salvado, K. Erlandsson, A. Bousse, P. van Mullekom, and B. F. Hutton, "Novel Collimation for Simultaneous SPECT/MRI," in 2014 IEEE NSS/MIC, Nov. 2014.

[10] D. Salvado, K. Erlandsson, A. Bousse, M. Occhipinti, P. Busca, C. Fiorini, and B. F. Hutton, "Collimator Design for a Brain SPECT/MRI Insert," IEEE Transactions on Nuclear Science, vol. 62, pp. 1716-24, Aug. 2015.

[11] D. Salvado, K. Erlandsson, and B. F. Hutton, "Shielding Requirements of a SPECT Insert for Installation in a PET/MRI System," in 2015 IEEE NSS/MIC, no. 4, Nov. 2015.

[12] K. Erlandsson, D. Salvado, A. Bousse, and B. Hutton, "Evaluation of a Partial Ring Design for the INSERT SPECT/MRI System," EJNMMI Physics, vol. 2, no. Suppl 1, p. A47, Jun. 2015. [Online]. Available: http://ejnmmiphys.springeropen.com/articles/10.1186/21977364-2-S1-A47

[13] J. Nuyts, K. Vunckx, M. Defrise, and C. Vanhove, "Small Animal Imaging with Multi-Pinhole SPECT," Methods, vol. 48, no. 2, pp. 83-91, Jun. 2009. [Online]. Available: http://linkinghub.elsevier.com/retrieve/pii/S1046202309000553

[14] L. R. Furenlid, D. W. Wilson, C. Yi-chun, K. Hyunki, P. J. Pietraski, M. J. Crawford, and H. H. Barrett, "FastSPECT II: A Second-Generation High-Resolution Dynamic SPECT Imager," IEEE Transactions on $\mathrm{Nu}$ clear Science, vol. 51, no. 3, pp. 631-5, Jun. 2004.

[15] F. van der Have, B. Vastenhouw, M. Rentmeester, and F. Beekman, "System Calibration and Statistical Image Reconstruction for Ultra-High Resolution Stationary Pinhole SPECT," IEEE Transactions on Medical Imaging, vol. 27, no. 7, pp. 960-71, Jul. 2008. [Online]. Available: http://ieeexplore.iee.org/document/4505843/

[16] B. W. Miller, R. Van Holen, H. H. Barrett, and L. R. Furenlid, "A System Calibration and Fast Iterative Reconstruction Method for Next-Generation SPECT Imagers," IEEE Transactions on Nuclear Science, vol. 59, no. 5, pp. 1990-6, Oct. 2012. [Online]. Available: http://ieeexplore.ieee.org/document/6213168/
[17] Y.-C. Chen, L. R. Furenlid, D. W. Wilson, and H. H. Barrett, "Calibration of Scintillation Cameras and Pinhole SPECT Imaging Systems," in Small-Animal SPECT Imaging, M. A. Kupinski and H. H. Barrett, Eds. Springer US, 2005, ch. 12, pp. 195-201.

[18] R. K. Rowe, J. N. Aarsvold, H. H. Barrett, J.-C. Chen, W. P. Klein, B. A. Moore, I. W. Pang, D. D. Patton, and T. A. White, "A Stationary Hemispherical SPECT Imager for Three-Dimensional Brain Imaging," The Journal of Nuclear Medicine, vol. 34, no. 3, pp. 474-80, 1993.

[19] D. Bequé, J. Nuyts, G. Bormans, P. Suetens, and P. Dupont, "Characterization of Acquisition Geometry of Pinhole SPECT," IEEE Transactions on Medical Imaging, vol. 22, pp. 599-612, 2003.

[20] D. Bequé, J. Nuyts, G. Bormans, P. Suetens, and P. Dupont, "Optimization of Pinhole SPECT Calibration," in 2003 IEEE NSS/MIC, vol. 4 2003, pp. 2343-7.

[21] M. Defrise, C. Vanhove, and J. Nuyts, "Perturbative Refinement of the Geometric Calibration in Pinhole SPECT," IEEE Transactions on Medical Imaging, vol. 27, no. 2, pp. 204-14, 2008.

[22] T. Ma, R. Yao, and Y. Shao, "Determination of Geometrical Parameters for Slit-Slat SPECT Imaging on MicroPET," in 2007 IEEE NSS/MIC, vol. 6, 2007, pp. 4285-8.

[23] Y. Shao, R. Yao, T. Ma, and P. Manchiraju, "Initial Studies of PETSPECT Dual-Tracer Imaging," in 2007 IEEE NSS/MIC, vol. 6, 2007, pp. 4198-204.

[24] R. Yao, X. Deng, T. Ma, J. Cadorette, Z. Cao, J.-F. Beaudoin, and R. Lecomte, "Geometrical Calibration for an Animal PET Converted SPECT," in 2010 IEEE NSS/MIC, 2010, pp. 3038-41.

[25] L. Cao and J. Peter, "Slit-Slat Collimator Equipped Gamma Camera for Whole-Mouse SPECT-CT Imaging," IEEE Transactions on Nuclear Science, vol. 59, no. 3, pp. 530-6, 2012.

[26] D. Salvado, K. Erlandsson, and B. F. Hutton, "Effects of the Collimator Magnification Factor in the Geometrical Calibration of SPECT Systems," in 2016 IEEE NSS/MIC, Nov. 2016.

[27] M. Occhipinti, P. Busca, and C. Fiorini, "Light Response Estimation and Gamma Events Reconstruction in Gamma Detectors Based on Continuous Scintillators," in 2016 IEEE NSS/MIC, Nov. 2016.

[28] A. Bousse, K. Erlandsson, N. Fuin, D. Salvado, and B. F. Hutton, "Variance Prediction in SPECT Reconstruction Based on the Fisher Information Using a Novel Angular Blurring Algorithm for Computation of the System Matrix," in 2013 IEEE NSS/MIC, 2013.

[29] E. J. Hoffman, P. D. Cutler, W. M. Digby, and J. C. Mazziotta, “3D Phantom to Simulate Cerebral Blood Flow and Metabolic Images for PET," IEEE Transactions on Nuclear Science, vol. 37, no. 2, pp. 61620, Apr. 1990. 